\title{
Activation Torque Estimation of Muscles by Forward Neural Networks (Forward-MuscleNET) for sEMG-Based Control of Assistive Robots
}

\author{
Ali Nasr ${ }^{1}$, Jiayuan He ${ }^{1}$, Ning Jiang ${ }^{1}$, and John McPhee ${ }^{1}$ \\ ${ }^{1}$ University of Waterloo \\ 200 University Ave W, Waterloo, Canada \\ a.nasr; jiayuan.he; ning.jiang; mcphee@uwaterloo.ca
}

\section{Extended Abstract}

Assistive devices are designed and manufactured to assist a disabled subject while recovering in daily living activities and productively in community life. To improve neuromuscular activity in rehabilitation and flexibility in power augmentation by assistive devices, the estimation of joint torque plays an essential role in volitional control. One method of joint torque estimation for controlling robotic exoskeletons (Exo)s is using biological signals, e.g. surface electromyogram (sEMG), which is measured along with voluntary muscle contraction [1]. However, from a physiological perspective, the relationship between sEMG and torque is considerably complicated. Although the Hill-type muscle model [2] is common, muscles within the model tolerate some deficiencies, e.g. complex musculoskeletal geometry, fitting muscle parameters, and actuator redundancy. The recent method is using a machine learning method which requires a significant amount of data (both kinematic and kinetic). We propose a potential solution to the difficulties associated with using machine learning.

In terms of decreasing the amount of data for training, we propose using a Muscle Torque Generator (MTG) model [3] or the Control-Oriented Muscle Torque (COMT) model as serial to the trained model. Since the MTG or COMT model consists of (1) passive torque, (2) torque-velocity scaling, (3) torque-angle scaling, and (4) activation torque, it can compensate the position/velocity dependence of muscle in the musculoskeletal system $(2,3)$ and the effects of viscoelastic structures surrounding the joint (1). The input of MTG or COMT is activation torque (4), and the output is joint torque.

In terms of efficient machine learning models, we propose the Forward-MuscleNET as a mapping model of sEMG to activation torque. The input of the Forward-MuscleNET has filtered sEMG signals of muscles (20-500 Hz Band-pass $\rightarrow 55-$ $65 \mathrm{~Hz}$ Band-Stop $\rightarrow$ Rectifying $\rightarrow 7 \mathrm{~Hz}$ Low-Pass $\rightarrow$ Normalize-2-SubjectMax). The output is calculated in three steps as follows: (i) motion capturing the kinematic data (position, velocity, and acceleration) and measuring the external load, (ii) solving the inverse dynamic model of skeletal (torque driven) model of the human joint by kinematic and kinetic data for finding the joint torque, and (iii) using MTG or COMT for calculating the activation torque by using the joint torque and kinematic data. The Forward-MuscleNET is a feedforward neural network with shallow configuration. By evaluating different configurations, we have found that the number of layers should be small, and the depth of each layer should be wide enough. Consequently, the performance, training time, and latency of the Forward-MuscleNET become better in comparison to the other configurations. In this study, we have mapped 11 channel sEMG signals of the shoulder muscles to the activation torque of the shoulder flexion/extension joint by two layers and 100 widths. The root mean squared error of the subject-specific (using one subject data) and the general (using 17 subjects' data) Forward-MuscleNET model were $0.1 \%$ and $23 \%$ of the activation torque, respectively. The higher error of the general model than the subject-specific model is reasonable due to differences in the anatomical configuration of subjects or differences in the attachment place of the sEMG. Finally, the subject-specific Forward-MuscleNET model will be used for joint torque estimation in controlling a robotic Exo.

\section{References}

[1] Kiguchi, Kazuo, and Yoshiaki Hayashi. "An EMG-based control for an upper-limb power-assist exoskeleton robot." IEEE Transactions on Systems, Man, and Cybernetics, vol. 42, no. 4, pp. 1064-1071, 2012.

[2] T. Siebert, C. Rode, W. Herzog, O. Till, and R. Blickhan, "Nonlinearities make a difference: comparison of two common Hill-type models with real muscle," Biological Cybernetics 98, no. 2, pp. 133-143, 2008. 
[3] K. Inkol, C. Brown, W. McNally, C. Jansen, and J. McPhee, "Muscle torque generators in multibody dynamic simulations of optimal sports performance," Multibody System Dynamics, 2020. 